ELECTRONIC RESEARCH ANNOUNCEMENTS

OF THE AMERICAN MATHEMATICAL SOCIETY

Volume 10, Pages 97-102 (August 31, 2004)

S $1079-6762(04) 00134-9$

\title{
A TRILINEAR RESTRICTION PROBLEM FOR THE PARABOLOID IN $\mathbb{R}^{3}$
}

\author{
JONATHAN BENNETT
}

(Communicated by Yitzhak Katznelson)

\begin{abstract}
We establish a sharp trilinear inequality for the extension operator associated to the paraboloid in $\mathbb{R}^{3}$. Our proof relies on a recent generalisation of the classical Loomis-Whitney inequality.
\end{abstract}

\section{INTRODUCTION}

Let $S$ be the paraboloid in $\mathbb{R}^{3}$ given by

$$
\left\{\left(\xi,|\xi|^{2}\right): \xi \in \mathbb{R}^{2}\right\}
$$

and let $d \sigma$ be the measure supported on $S$ given by

$$
\int \phi d \sigma=\int_{\mathbb{R}^{2}} \phi\left(\xi,|\xi|^{2}\right) d \xi
$$

For $g \in L^{1}(d \sigma)$, we define the extension operator applied to $g$ to be

$$
\widehat{g d \sigma}(x)=\int e^{-i x \cdot y} g(y) d \sigma(y) .
$$

The classical restriction conjecture (for the paraboloid in this case) proposes the exponents $p$ and $q$ for which this operator is bounded from $L^{p}(d \sigma)$ to $L^{q}\left(\mathbb{R}^{3}\right)$ - see [4].

It has long been known that certain $L^{p}-L^{q}$ estimates of this type have what are often referred to as "bilinear improvements". In particular, the range of $p$ 's and $q$ 's for which the bilinear operator

$$
(f, g) \mapsto \widehat{f d \sigma} \widehat{g d \sigma}
$$

maps $L^{p} \times L^{p}$ to $L^{q}$, for $f$ and $g$ satisfying a certain "support separation" condition, is wider than that which is directly predicted by Hölder's inequality and the restriction conjecture. For a detailed description of these notions see 5 .

The purpose of this note is to bring to light certain natural trilinear estimates in this context.

Received by the editors December 18, 2003 and, in revised form, July 16, 2004.

2000 Mathematics Subject Classification. Primary 42B10.

Key words and phrases. Multilinear estimates, Fourier extension operator.

The author was supported by an EPSRC Postdoctoral Fellowship. 
Theorem 1.1. Suppose $P_{1}, P_{2}, P_{3} \in S$ are such that the normals to $S$ at these points span $\mathbb{R}^{3}$. Then there exist neighbourhoods $U_{1}, U_{2}, U_{3} \subset S$ of $P_{1}, P_{2}, P_{3}$ respectively, and a constant $C$ such that

$$
\|\widehat{f d \sigma} \widehat{g d \sigma} \widehat{h d \sigma}\|_{L^{2}\left(\mathbb{R}^{3}\right)} \leq C\|f\|_{4 / 3}\|g\|_{4 / 3}\|h\|_{4 / 3}
$$

for all $f, g, h \in L^{4 / 3}(d \sigma)$ satisfying

$$
\operatorname{supp}(f) \subset U_{1}, \quad \operatorname{supp}(g) \subset U_{2}, \quad \text { and } \quad \operatorname{supp}(h) \subset U_{3} .
$$

\section{Remarks.}

(1) The exponent $4 / 3$ on the right hand side is sharp given the exponent 2 on the left. Naturally, the estimate fails to hold if the points $P_{1}, P_{2}, P_{3}$ are separated in a more naive way. In particular, if we merely ask that the points $P_{1}, P_{2}, P_{3}$ are distinct, the best $L^{2}$ estimate possible is with $4 / 3$ replaced by $18 / 13$. This can be seen as a consequence of the bilinear analysis in [5]. Furthermore, if we place no restriction at all on the points $P_{1}, P_{2}, P_{3}$, the best $L^{2}$ estimate is with $18 / 13$ replaced by $3 / 2$. This follows easily from the existing linear restriction theory (see [4]).

(2) On a technical level, our approach is related to the $12 / 7$ bilinear restriction inequality of Moyua, Vargas and Vega [3] (see also [5]). Inherent in their estimate is a bound for a certain linear Radon transform in the plane. In the trilinear setting matters are different partly because the Radon-like transforms that arise are bilinear.

(3) Theorem 1.1 was originally inspired by a multilinear inequality for certain spherical averages of the extension operator (this time associated to the sphere) - see [1].

(4) It seems plausible that multilinear restriction estimates of this nature might have a role to play in proving new linear restriction theorems in dimensions 3 and above. One only needs to glance at $[5]$ to imagine this.

The key ingredient in our proof of Theorem 1.1 is the following generalisation of the classical Loomis-Whitney inequality (see [2] for a proof of this).

Lemma 1.2. If $\pi_{1}, \pi_{2}, \pi_{3}: \mathbb{R}^{3} \rightarrow \mathbb{R}^{2}$ are submersions in a neighbourhood of $x_{0} \in \mathbb{R}^{3}$ such that the kernels of $d \pi_{1}\left(x_{0}\right), d \pi_{2}\left(x_{0}\right)$, and $d \pi_{3}\left(x_{0}\right)$ span $\mathbb{R}^{3}$, then for all cut-off functions a supported in a sufficiently small neighbourhood of $x_{0}$, there is a constant $C$ such that

$$
\int_{\mathbb{R}^{3}} f\left(\pi_{1}(x)\right) g\left(\pi_{2}(x)\right) h\left(\pi_{3}(x)\right) a(x) d x \leq C\|f\|_{2}\|g\|_{2}\|h\|_{2}
$$

for all $f, g, h \in L^{2}\left(\mathbb{R}^{2}\right)$.

\section{The Proof of Theorem 1.1}

Let $u, v, w \in \mathbb{R}^{2}$ be such that $P_{1}=\left(u,|u|^{2}\right), P_{2}=\left(v,|v|^{2}\right)$ and $P_{3}=\left(w,|w|^{2}\right)$. It is easily seen that the hypothesis on the points $P_{1}, P_{2}$ and $P_{3}$ is equivalent to the non-colinearity of the points $u, v$ and $w$ in $\mathbb{R}^{2}$. By Plancherel's theorem, symmetry and multilinear interpolation it suffices to prove that there exist neighbourhoods 
$\Omega_{1}, \Omega_{2}, \Omega_{3} \subset \mathbb{R}^{2}$ of $u, v, w$, and a constant $C$ such that

$$
\begin{aligned}
\int_{\left(\mathbb{R}^{2}\right)^{6}} f_{1}(x) & g_{1}(y) h_{1}(z) f_{2}\left(x^{\prime}\right) g_{2}\left(y^{\prime}\right) h_{2}\left(z^{\prime}\right) \\
\times & \delta\left(|x|^{2}+|y|^{2}+|z|^{2}-\left|x^{\prime}\right|^{2}-\left|y^{\prime}\right|^{2}-\left|z^{\prime}\right|^{2}\right) \\
& \times \delta\left(x+y+z-x^{\prime}-y^{\prime}-z^{\prime}\right) d x d y d z d x^{\prime} d y^{\prime} d z^{\prime} \\
\leq C & \left\{\begin{array}{l}
\left\|f_{1}\right\|_{2}\left\|g_{1}\right\|_{2}\left\|h_{1}\right\|_{2}\left\|f_{2}\right\|_{1}\left\|g_{2}\right\|_{1}\left\|h_{2}\right\|_{1} \\
\left\|f_{1}\right\|_{2}\left\|g_{1}\right\|_{2}\left\|h_{1}\right\|_{1}\left\|f_{2}\right\|_{1}\left\|g_{2}\right\|_{1}\left\|h_{2}\right\|_{2}
\end{array}\right.
\end{aligned}
$$

for all

$$
\operatorname{supp}\left(f_{i}\right) \subset \Omega_{1}, \quad \operatorname{supp}\left(g_{i}\right) \subset \Omega_{2}, \quad \text { and } \quad \operatorname{supp}\left(h_{i}\right) \subset \Omega_{3} .
$$

(Note that $f_{1}, g_{1}, h_{1}, f_{2}, g_{2}$ and $h_{2}$ are now functions on $\mathbb{R}^{2}$ rather than $S$.)

The proofs of the two inequalities in (2.1) follow the same general scheme. We begin with the second as it is slightly more straightforward algebraically. It should be remarked that in order to prove Theorem 1.1 for characteristic functions it is enough to obtain just one of these inequalities.

Since $h_{1}, f_{2}$ and $g_{2}$ are controlled in $L^{1}$, we may suppose that $h_{1}=\delta_{z}, f_{2}=\delta_{x^{\prime}}$ and $g_{2}=\delta_{y^{\prime}}$ for some $\left(z, x^{\prime}, y^{\prime}\right)$ in a sufficiently small neighbourhood of $(w, u, v)$. Writing $X=x-x^{\prime}$ and $Y=y-y^{\prime}$, the left hand side of the above becomes

$$
\begin{aligned}
& \int f_{1}\left(X+x^{\prime}\right) g_{1}\left(Y+y^{\prime}\right) h_{2}(X+Y+z) \\
& \quad \times \delta\left(\left|X+x^{\prime}\right|^{2}+\left|Y+y^{\prime}\right|^{2}+|z|^{2}-\left|x^{\prime}\right|^{2}-\left|y^{\prime}\right|^{2}-|X+Y+z|^{2}\right) d X d Y \\
&=\frac{1}{2} \int f_{1}\left(X+x^{\prime}\right) g_{1}\left(Y+y^{\prime}\right) h_{2}(X+Y+z) \\
& \times \delta\left(\left(x^{\prime}-z\right) \cdot X+\left(y^{\prime}-z\right) \cdot Y-X \cdot Y\right) d X d Y .
\end{aligned}
$$

By the translation invariance of $L^{p}$-norms, it suffices to prove that

$$
\int f(X) g(Y) h(X+Y) \delta\left(\left(x^{\prime}-z\right) \cdot X+\left(y^{\prime}-z\right) \cdot Y-X \cdot Y\right) d X d Y
$$

is bounded by $C\|f\|_{2}\|g\|_{2}\|h\|_{2}$, for all $f, g$, and $h$ supported in sufficiently small neighbourhoods of the origin.

By translation (or Galilean) invariance, scaling and a rotation, we may suppose that $z=0, x^{\prime}=e_{1}$ and $y^{\prime}=a$, where $a_{2}>c$ for some constant $c>0$ depending on $u, v$, and $w$. Since

$$
e_{1} \cdot X+a \cdot Y-X \cdot Y=\left(1-Y_{1}\right)\left(X_{1}-\frac{X_{2} Y_{2}-a \cdot Y}{1-Y_{1}}\right),
$$

we are reduced to proving that for some neighbourhood $U$ of the origin in $\mathbb{R}^{3}$,

$$
\int_{U} f\left(\pi_{1}\left(X_{2}, Y\right)\right) g\left(\pi_{2}\left(X_{2}, Y\right)\right) h\left(\pi_{3}\left(X_{2}, Y\right)\right) d X_{2} d Y \leq C\|f\|_{2}\|g\|_{2}\|h\|_{2},
$$

whereำ

$$
\pi_{1}\left(X_{2}, Y\right)=\left(\frac{X_{2} Y_{2}-a \cdot Y}{1-Y_{1}}, X_{2}\right), \quad \pi_{2}\left(X_{2}, Y\right)=Y
$$

\footnotetext{
${ }^{1}$ In deriving this representation of the trilinear form we have used the fact that for $Y$ in a sufficiently small neighbourhood of the origin, $1-Y_{1}$ is bounded away from 0 .
} 
and $\pi_{3}=\pi_{1}+\pi_{2}$. (As may be expected, there are other parametrisations that may be chosen here.)

In order to prove (2.3) we appeal to Lemma 1.2. After a straightforward computation we see that $\pi_{1}, \pi_{2}$, and $\pi_{3}$ are submersions in a neighbourhood of 0 , and furthermore,

$$
\begin{gathered}
\operatorname{ker} d \pi_{1}(0)=\left\langle\left(0,-a_{2}, a_{1}\right)\right\rangle, \\
\operatorname{ker} d \pi_{2}(0)=\langle(1,0,0)\rangle,
\end{gathered}
$$

and

$$
\text { ker } d \pi_{3}(0)=\left\langle\left(1-a_{1},-a_{2}, a_{1}-1\right)\right\rangle .
$$

Since the determinant of the above three generators is equal to $a_{2}>c>0$, (2.3) follows.

We now turn to the proof of the first inequality in (2.1). Since $f_{2}, g_{2}$, and $h_{2}$ are controlled in $L^{1}$, we may suppose that $f_{2}=\delta_{x^{\prime}}, g_{2}=\delta_{y^{\prime}}$ and $h_{2}=\delta_{z^{\prime}}$ for some $\left(x^{\prime}, y^{\prime}, z^{\prime}\right)$ in a sufficiently small neighbourhood of $(u, v, w)$. Again, by Galilean invariance, scaling, and a rotation, we may suppose that $z^{\prime}=0, x^{\prime}=e_{1}$ and $y^{\prime}=a$, where $a_{2}>c$ for some constant $c>0$ depending on $u, v$, and $w$. Writing $X=x-x^{\prime}$ and $Y=y-y^{\prime}$, the left hand side of (2.1) becomes

$$
\begin{aligned}
& \int f_{1}\left(X+x^{\prime}\right) g_{1}\left(Y+y^{\prime}\right) h_{1}\left(-X-Y+z^{\prime}\right) \\
& \quad \times \delta\left(\left|X+x^{\prime}\right|^{2}+\left|Y+y^{\prime}\right|^{2}+\left|X+Y-z^{\prime}\right|^{2}-\left|x^{\prime}\right|^{2}-\left|y^{\prime}\right|^{2}-\left|z^{\prime}\right|^{2}\right) d X d Y \\
&=\frac{1}{2} \int f_{1}\left(X+x^{\prime}\right) g_{1}\left(Y+y^{\prime}\right) h_{1}(-X-Y) \\
& \quad \times \delta\left(\left|X+\left(e_{1}+Y\right) / 2\right|^{2}-\left|e_{1}+Y\right|^{2} / 4+|Y|^{2}+a \cdot Y\right) d X d Y
\end{aligned}
$$

It thus suffices to prove that

$$
\begin{aligned}
\int f(X) & g(Y) h(X+Y) \\
\quad \times & \delta\left(\left|X+\left(e_{1}+Y\right) / 2\right|^{2}-\left|e_{1}+Y\right|^{2} / 4+|Y|^{2}+a \cdot Y\right) d X d Y
\end{aligned}
$$

is bounded by $C\|f\|_{2}\|g\|_{2}\|h\|_{2}$, for all $f, g$, and $h$ supported in sufficiently small neighbourhoods of the origin.

Now for fixed $Y, X$ lives on the circle given parametrically by

$$
X=-\frac{1}{2}\left(e_{1}+Y\right)+r(Y)(\cos t, \sin t),
$$

where $r(Y)^{2}=\frac{1}{4}\left|e_{1}+Y\right|^{2}-|Y|^{2}-a \cdot Y$ and $t \in \mathbb{R}$. Observe that since we are only concerned with $X$ and $Y$ in a small neighbourhood of the origin, we need only consider $t$ in a small neighbourhood of 0 . Hence we are reduced to proving that for some neighbourhood $U$ of the origin in $\mathbb{R}^{3}$,

$$
\int_{U} f\left(\pi_{1}(Y, t)\right) g\left(\pi_{2}(Y, t)\right) h\left(\pi_{3}(Y, t)\right) d Y d t \leq C\|f\|_{2}\|g\|_{2}\|h\|_{2},
$$

where光

$$
\begin{gathered}
\pi_{1}(Y, t)=Y \\
\pi_{2}(Y, t)=-\left(e_{1}+Y\right) / 2+r(Y)(\cos t, \sin t),
\end{gathered}
$$

\footnotetext{
${ }^{2}$ In deriving this representation we have used the fact that for $Y$ in a sufficiently small neigbourhood of the origin, $r(Y)$ is bounded away from 0 .
} 
and $\pi_{3}=\pi_{1}+\pi_{2}$. After a straightforward computation we see that $\pi_{1}, \pi_{2}$, and $\pi_{3}$ are submersions in a neighbourhood of 0 , and furthermore,

$$
\begin{gathered}
\operatorname{ker} d \pi_{1}(0)=\langle(0,0,1)\rangle, \\
\operatorname{ker} d \pi_{2}(0)=\left\langle\left(-a_{2}, a_{1}, a_{1}\right)\right\rangle,
\end{gathered}
$$

and

$$
\operatorname{ker} d \pi_{3}(0)=\left\langle\left(-a_{2}, a_{1}-1,1-a_{1}\right)\right\rangle .
$$

As before, the determinant of the above three generators is equal to $a_{2}>c>0$, and so (2.4) follows.

Remark. There is a minor technical issue in our argument that we have glossed over here. It is of course important that the neighbourhoods of the origin and the constant $C$ appearing in (2.2) may be chosen independently of $\left(z, x^{\prime}, y^{\prime}\right)$ belonging to a sufficiently small neighbourhood of $(w, u, v)$. This detail may be easily dealt with by appealing to a more quantative version of Lemma 1.2 (such as that in 2]). On doing this, one may also quantify the constant $C$ and the neighbourhoods $U_{1}, U_{2}$, and $U_{3}$ appearing in the statement of Theorem 1.1. These issues will be elucidated in a subsequent paper.

\section{THE WIDER CONTEXT}

The standard examples (see [4]) in the context of the restriction conjecture suggest the following $n$-linear conjecture in $n$ dimensions. Here $S$ will denote the paraboloid

$$
\left\{\left(\xi,|\xi|^{2}\right): \xi \in \mathbb{R}^{n-1}\right\}
$$

in $\mathbb{R}^{n}$, and $d \sigma$ the measure supported on $S$ given by

$$
\int \phi d \sigma=\int_{\mathbb{R}^{n-1}} \phi\left(\xi,|\xi|^{2}\right) d \xi
$$

Conjecture 3.1. Suppose $P_{1}, \ldots, P_{n} \in S$ are such that the normals to $S$ at these points span $\mathbb{R}^{n}$. Then there exist neighbourhoods $U_{1}, \ldots, U_{n} \subset S$ of $P_{1}, \ldots, P_{n}$ respectively, and a constant $C$ such that

$$
\left\|\prod_{j=1}^{n} \widehat{g_{j} d \sigma}\right\|_{L^{q / n}\left(\mathbb{R}^{n}\right)} \leq C \prod_{j=1}^{n}\left\|g_{j}\right\|_{p}
$$

for all $g_{j} \in L^{p}(d \sigma)$ satisfying

$$
\operatorname{supp}\left(g_{j}\right) \subset U_{j}, \quad 1 \leq j \leq n,
$$

if and only if $q \geq \frac{2 n}{n-1}$ and $p^{\prime} \leq \frac{n-1}{n} q$.

We remark that by interpolation and Hölder's inequality, this conjecture is equivalent to the inequality

$$
\left\|\prod_{j=1}^{n} \widehat{g_{j} d \sigma}\right\|_{L^{2 /(n-1)\left(\mathbb{R}^{n}\right)}} \leq C \prod_{j=1}^{n}\left\|g_{j}\right\|_{2} .
$$




\section{Remarks.}

(1) The known linear and bilinear restriction theory clearly implies progress on the above conjecture, just by Hölder's inequality. As may be expected, the non-trivial exponents obtained in this way lie away from the sharp line $p^{\prime}=\frac{n-1}{n} q$. The purpose of Theorem 1 is to provide a non-trivial point on this line in three dimensions.

(2) The above conjecture may in fact be made for quite general smooth codimension 1 submanifolds of $\mathbb{R}^{n}$. The proof of Theorem 1.1 may also be adapted to this general context. The details of this will be made explicit in a subsequent paper.

(3) By a standard Rademacher function argument, the above conjecture (3.1) implies a certain multilinear Kakeya-type estimate, which we now describe. Merely for expositional convenience we replace the paraboloid with the unit sphere here. Suppose that the vectors $\omega_{1}, \ldots, \omega_{n} \in \mathbb{S}^{n-1}$ span $\mathbb{R}^{n}$, then there exist neighbourhoods $U_{1}, \ldots, U_{n} \subset \mathbb{S}^{n-1}$ of $\omega_{1}, \ldots, \omega_{n}$ respectively, and a constant $C$ such that

$$
\left\|\sum_{T_{1} \in \mathbb{T}_{1}} \chi_{T_{1}} \sum_{T_{2} \in \mathbb{T}_{2}} \chi_{T_{2}} \cdots \sum_{T_{n} \in \mathbb{T}_{n}} \chi_{T_{n}}\right\|_{L^{1 /(n-1)}\left(\mathbb{R}^{n}\right)} \leq C \sum_{T_{1} \in \mathbb{T}_{1}}\left|T_{1}\right| \cdots \sum_{T_{n} \in \mathbb{T}_{n}}\left|T_{n}\right|
$$

for all families $\mathbb{T}_{1}, \ldots, \mathbb{T}_{n}$ of $\delta \times \cdots \times \delta \times 1$-tubes in $\mathbb{R}^{n}$ such that their directions belong to $U_{1}, \ldots, U_{n}$ respectively. Here $C$ should be independent of the small parameter $0<\delta \leq 1$. We refer the reader to [5] for a discussion of the linear and bilinear Kakeya phenomena.

(4) It might be interesting to establish whether Theorem 1.1 may be generalised to $n \geq 4$. It is not immediately clear how our approach may be extended since the multilinear Radon transforms that arise cannot be dealt with directly by the natural higher-dimensional version of Lemma 1.2 .

\section{ACKNOWLEDGEMENTS}

We would like to thank Tony Carbery, Susana Gutiérrez and Jim Wright for a number of very useful discussions on the subject of this note.

\section{REFERENCES}

1. J. A. Barceló, J. M. Bennett, and A. Carbery, A multilinear extension inequality in $\mathbb{R}^{n}$, Bull. London Math. Soc. 36 (3) (2004), 407-412. MR2038728

2. J. M. Bennett, A. Carbery, and J. Wright, A generalisation of the Loomis-Whitney inequality in $\mathbb{R}^{n}$, in preparation.

3. A. Moyua, A. Vargas, L. Vega, Restriction theorems and maximal operators related to oscillatory integrals in $\mathbb{R}^{3}$, Duke Math. J. 96 (3) (1999), 547-574. MR 1671214 (2000b:42017)

4. E. M. Stein, Harmonic Analysis, Princeton University Press, Princeton, NJ, 1993. MR 1232192 (95c:42002)

5. T. Tao, A. Vargas, L. Vega, A bilinear approach to the restriction and Kakeya conjectures, J. Amer. Math. Soc. 11 (1998), 967-1000. MF1625056 (99f:42026)

School of Mathematics, JCMB, Kings Buildings, Mayfield Road, Edinburgh, EH9 3JZ, SCOTLAND

E-mail address: J.Bennett@ed.ac.uk 